\title{
USE OF JEFFERSON LAB'S HIGH AVERAGE POWER FEL AS A THOMSON BACKSCATTER X-RAY SOURCE
}

\author{
G. A. Krafft, TJNAF, 12000 Jefferson Ave., Newport News, VA 23606
}

\section{Abstract}

Recently, workers from Berkeley have measured Thomson forward scattered X-rays at the ALS injector. The average power of their device was limited by, among other things, the repetition rate of the high power laser. In order to achieve high average power, it is natural to investigate the possibility of backscatter from the recirculating optical pulses in Jefferson Lab's CW IRFEL, now under construction. Photon brightnesses of order $10^{7}$ photons $/\left(\mathrm{sec} \mathrm{mm}^{2}\right.$ $\operatorname{mrad}^{2}$ ) will be produced at this facility, with a spectrum extending up to $7 \mathrm{keV}$ in energy, in the present operating mode. A near-term upgrade to $75 \mathrm{MeV}$ yields about 30 $\mathrm{keV}$ photons with similar brightness. In the longer term, the UV upgrade of our device could potentially produce 4 $\mathrm{MeV}$ photons with substantially higher brightness. Scaling laws for such configurations are given and these three cases are presented in detail.

\section{X-RAY LUMINOSITY}

In the Berkeley work [1], and in the current work, the primary purpose of the experiments is to produce short-timeduration pulses. Such pulses may be produced by scattering a short IR laser pulse at ninety degrees to a $45 \mathrm{MeV}$ electron beam that has been focussed transversely to a small spot size, or by 180 degree scattering off a very short bunch. A distinguishing feature of the IRFEL at Jefferson Lab is its relatively short bunch duration of 1 psec rms; the radiation pulse distribution mirrors the "micropulse current" distribution of the beam in time.

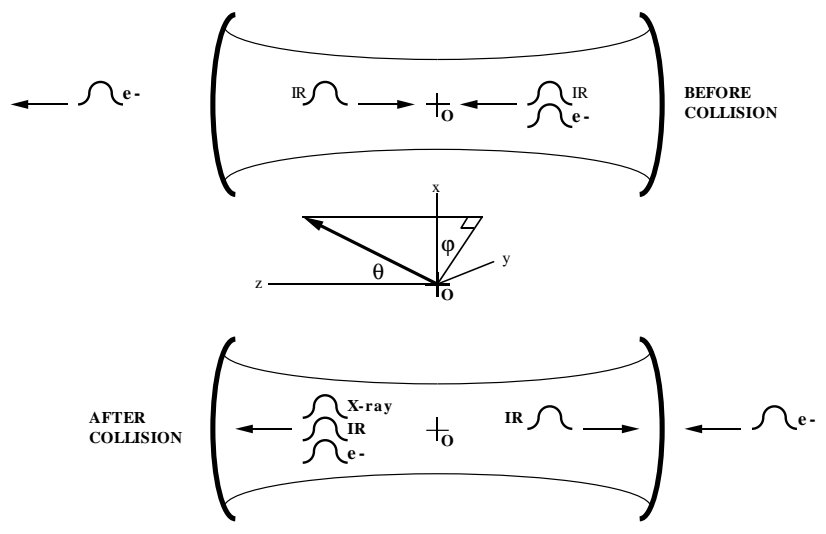

Figure 1: Scattering Geometry

In Fig. (1) the general geometry is presented. The micropulse separation is chosen to be equal to the optical cavity length; two optical pulses are circulating in the optical cavity. Before the collision, overlapping optical and electron pulses move downstream, and an optical pulse moves upstream. After the collision, overlapping optical, electron, and high energy photon pulses move downstream, and again, the scattered pulse continues upstream. There are clearly $f=c / l$ collisions per unit time, where $c$ is the velocity of light and $l$ is the optical cavity length. To get the total power, the total energy transferred into high energy photons in one collision should be estimated.

To analyze a single collision, recall that the total number of scattered X-rays is a Lorentz invariant quantity, as is $\sigma$, the total scattering cross section in the rest frame of the electrons [2]. Now $\Phi_{e}^{\mu}=J^{\mu} / e$ is a 4-vector, where $J^{\mu}$ is the usual current density 4-vector, whose time component is the number density of electrons. Likewise $\Phi_{p}^{\mu}=\left(n_{p}, n_{p} \beta_{p}\right)$ is the 4-vector describing the photon flux. Clearly, $N_{s}$, the number of scattered photons is proportional to both $n_{p}$ and $n_{e}$, and $d^{4} x$ is Lorentz invariant. Consequently $N_{s}$ must be expressed as

$$
\begin{gathered}
N_{s}=\int \Phi_{p \mu} \Phi_{e}^{\mu} \sigma d^{4} x \\
=\int\left(1-\beta_{p} \cdot \beta_{e}\right) n_{p}(x, y, z, t) n_{e}(x, y, z, t) \sigma d^{4} x .
\end{gathered}
$$

If the distributions are separable, $n_{p}(x, y, z, t)=$ $n_{p}(x, y) I_{p}(z+c t), n_{e}(x, y, z, t)=n_{e}(x, y) I_{e}(z-\beta c t)$, where $I_{p}, I_{e}$ are unit normalized and where $n_{p}(x, y)$ and $n_{e}(x, y)$ are the transverse number distribution of the lowenergy photons and electrons respectively, the above integral simplifies to

$$
N_{s}=\int n_{p}(x, y) n_{e}(x, y) \sigma_{T} d x d y .
$$

The total Thomson scattering cross section, $\sigma_{T}=8 \pi r_{e}^{2} / 3$, is the cross section for the process of interest, $\beta=v_{z} / c$ is the usual relativistic velocity, and $r_{e}$ is the classical electron radius. Specializing to the case of overlapped Gaussians of the same rms size $\sigma$, one obtains

$$
N_{s}=\frac{N_{p} N_{e}}{4 \pi \sigma^{2}} \sigma_{T}
$$

where $N_{p}$ is the number of low-energy photons and $N_{e}$ is the number of electron scatterers. One defines a "luminosity"

$$
L=f \frac{N_{p} N_{e}}{4 \pi \sigma^{2}}
$$

which defines the intensity generated by the scattering geometry. Such a quantity may be used to evaluate the geometry, much as the usual definition of luminosity is used in 
particle physics. Typical values fall in the $10^{33} \mathrm{~cm}^{-2} \mathrm{sec}^{-1}$ range, for the types of devices that we will consider. Also note that as $N_{p}$ tends to be proportional to $N_{e}$ in an FEL oscillator geometry, luminosity can scale as rapidly as $N_{e}^{2}$ (i. e., as the beam current squared) if the electron and photon beam sizes remain the same as the current is changed. To estimate the total X-ray power multiply $L \sigma_{T}$ by the energy of a typical photon.

\section{PROPERTIES OF THE RADIATION}

Next, the properties of the backscattered radiation are discussed. As in figure 1, laboratory quantities are referred to a coordinate system with $z$-axis in the beam direction. The $x$-axis is taken to be in the (vertical) polarization direction of the circulating laser pulse. The $y$-axis, into the paper, completes the right-hand set. The origin of the coordinate system is the point of collision; standard polar coordinates $\theta$ and $\phi$ are used to describe the direction of the backscattered radiation. A beam-fixed primed frame is also used, with unit vectors in the same directions as in the laboratory system, and with similar polar coordinates $\theta^{\prime}$ and $\phi^{\prime}$. The beam relativistic factors are $\beta=v_{z} / c$ and $\gamma=1 / \sqrt{1-\beta^{2}}$. The energy of the low-energy photons in the laboratory frame will be denoted $E_{0}$.

In the beam frame, the upshifted energy of the photons $E^{\prime}=\gamma(1+\beta) E_{0}$ is much less than the electron rest energy, and the scattering may be described by the Thomson scattering cross section [3],

$$
\frac{d \sigma_{T}^{\prime}}{d \Omega^{\prime}}=\frac{d \sigma_{T \|}^{\prime}}{d \Omega^{\prime}}+\frac{d \sigma_{T \perp}^{\prime}}{d \Omega^{\prime}}
$$

where

$$
\frac{d \sigma_{T \|}^{\prime}}{d \Omega^{\prime}}=r_{e}^{2} \cos ^{2} \theta^{\prime} \cos ^{2} \phi^{\prime}
$$

is the scattering into the polarization parallel to the plane of scattering,

$$
\frac{d \sigma_{T \perp}^{\prime}}{d \Omega^{\prime}}=r_{e}^{2} \sin ^{2} \phi^{\prime}
$$

is the scattering into the polarization perpendicular to the plane of scattering, and $r_{e}$ is the classical electron radius. The total cross section is $\sigma_{T}=8 \pi r_{e}^{2} / 3$, with 3 times the intensity in the perpendicular polarization than in the parallel polarization. These cross sections are used to calculate the total number of photons produced per collision in a given solid angle

$$
\begin{aligned}
\frac{d N_{s \|}}{d \Omega^{\prime}} & =\frac{N_{p} N_{e}}{4 \pi \sigma^{2}} \frac{d \sigma_{T \|}^{\prime}}{d \Omega^{\prime}} \\
\frac{d N_{s \perp}}{d \Omega^{\prime}} & =\frac{N_{p} N_{e}}{4 \pi \sigma^{2}} \frac{d \sigma_{T \perp}^{\prime}}{d \Omega^{\prime}}
\end{aligned}
$$

Now, the distribution must be translated back into the laboratory frame. The scattering angles transform as

$$
\begin{gathered}
\cos \theta^{\prime}=\frac{\cos \theta-\beta}{1-\beta \cos \theta} \\
\phi^{\prime}=\phi .
\end{gathered}
$$

The cross sections are converted by noting that the total number of scattered photons must be Lorentz invariant. Therefore

$$
\frac{d \sigma}{d \Omega}(\theta, \phi)=\frac{d \sigma^{\prime}}{d \Omega^{\prime}}\left(\theta^{\prime}(\theta), \phi^{\prime}(\phi)\right) \frac{d \Omega^{\prime}}{d \Omega},
$$

and so

$$
\begin{gathered}
\frac{d \sigma_{T \|}}{d \Omega}(\theta, \phi)=r_{e}^{2}\left(\frac{\cos \theta-\beta}{1-\beta \cos \theta}\right)^{2} \frac{\cos ^{2} \phi}{\gamma^{2}(1-\beta \cos \theta)^{2}} \\
\frac{d \sigma_{T \perp}}{d \Omega}(\theta, \phi)=r_{e}^{2} \frac{\sin ^{2} \phi}{\gamma^{2}(1-\beta \cos \theta)^{2}} .
\end{gathered}
$$

These cross-sections have the characteristic opening angle of $1 / \gamma$ for the majority of the scattered radiation.

The total intensity is calculated by multiplying the number of scatters per collision by the collision frequency and photon energy. In general, the energy of the back-scattered photons is

$$
E=\gamma^{2}(1+\beta)\left(1+\beta \cos \theta^{\prime}\right) E_{0}=\frac{(1+\beta) E_{0}}{1-\beta \cos \theta},
$$

and the scattering intensity, in power per unit solid angle, is

$$
\begin{gathered}
\frac{d P_{\|}}{d \Omega}=L \frac{(1+\beta) E_{0}}{1-\beta \cos \theta} \frac{d \sigma_{T \|}}{d \Omega} \\
\frac{d P_{\perp}}{d \Omega}=L \frac{(1+\beta) E_{0}}{1-\beta \cos \theta} \frac{d \sigma_{T \perp}}{d \Omega} .
\end{gathered}
$$

Alternatively, these results are obtained from the standard synchrotron radiation integrals; I have not been able to find these expressions in such a simple form in the extant literature.

The obvious generalization of Eqs. (1) to include nonzero electron and optical beam angles is to convolve over the (unit normalized and zero centered) relative angle distribution of the two beams. The generalization to include finite pulse effects is also straightforward:

$$
\begin{gathered}
\frac{d P_{\|}}{d \omega d \Omega}=\frac{f c}{4 \pi^{2}}\left|\tilde{E}\left(\frac{\omega(1-\beta \cos \theta)}{(1+\beta)}\right)\right|^{2} \frac{d \sigma_{T \|}}{d \Omega} \\
\frac{d P_{\perp}}{d \omega d \Omega}=\frac{f c}{4 \pi^{2}}\left|\tilde{E}\left(\frac{\omega(1-\beta \cos \theta)}{(1+\beta)}\right)\right|^{2} \frac{d \sigma_{T \perp}}{d \Omega},
\end{gathered}
$$

where $\tilde{E}(\omega)=\int E(t) e^{i \omega t} d t$ is the Fourier transform of the incident electric field of the low-energy photons. As there are about 40 oscillations in the micropulse, the energy spread in the emerging radiation is about $2 \%$ at each observation angle. The maximum energy of the scattered photons, at $\theta=0$, is

$$
E_{\max }=\gamma^{2}(1+\beta)^{2} E_{0} \approx 4 \gamma^{2} E_{0} .
$$




$\begin{array}{lccccccc} & \text { Phase I } & \text { Phase II } & \text { Phase III } & \text { Berkeley } & \text { NRL } & \text { APS } & \text { Units } \\ E_{\text {beam }} & 42 & 75 & 200 & 50 & 50 & 6000 & \mathrm{MeV} \\ E_{\text {photons }} & 6 & 70 & 3800 & 30\left(90^{\circ}\right) & 47 & 10-100 & \mathrm{keV} \\ E_{\text {Ophotons }} & 0.21 & 0.8 & 6.2 & 1.5 & 1.2 & \mathrm{NA} & \mathrm{eV} \\ f & 37.5 \mathrm{M} & 37.5 \mathrm{M} & 37.5 \mathrm{M} & 2 & 1 \mathrm{k} & 0.1-1 \mathrm{G} & \mathrm{Hz} \\ \sigma_{x} & 500 & 400 & 200 & 50 & 50 & 300 \times 90 & \mu \mathrm{m} \\ \sigma_{t} & 1000 & 1000 & 1000 & 100 & 1000 & 20,000 & \mathrm{fsec} \\ q & 135 & 135 & 135 & (0.05) 1300 & 1000 & \mathrm{NA} & \mathrm{pC} \\ N_{p} & 8.0 \times 10^{15} & 3.4 \times 10^{15} & 5.4 \times 10^{14} & 2.4 \times 10^{17} & 1.0 \times 10^{19} & \mathrm{NA} & \\ L & 8.1 \times 10^{37} & 5.3 \times 10^{37} & 2.1 \times 10^{37} & 6.2 \times 10^{33} & 2.0 \times 10^{39} & \mathrm{NA} & \mathrm{m}^{-2} \mathrm{sec}^{-1} \\ \text { Brightness } & 2.2 \times 10^{7} & 7.2 \times 10^{7} & 5.2 \times 10^{8} & 2.4 \times 10^{5} & 7.8 \times 10^{10} & 5 \times 10^{19} & \mathrm{~mm}^{-2} \mathrm{mrad}^{-2} \mathrm{sec}^{-1}\end{array}$

Table 1: X-ray source parameters for various backscatter sources

\section{RESULTS}

The accelerator parameters of Jefferson Lab's IRFEL (IR Demo) project are given in another contribution to this conference [4]. Table 1 presents the expected X-ray brightness from the IR Demo project (Phase I), and two potential upgrade projects: a near-term upgrade to $75 \mathrm{MeV}$ yielding about $1 \mu \mathrm{m}$ radiation circulating in the FEL (Phase II), and a longer-term upgrade to $200 \mathrm{MeV}$ yielding UV light in the FEL (Phase III). For comparison purposes, the reported performance in the Berkeley measurements, a design patterned on early Naval Research Laboratory (NRL) work on this topic [5], and from a typical high energy storage ring, the Advanced Photon Source (APS), are also given.

Compared to the Berkeley proposal, the Phase I IRFEL at Jefferson Lab will produce photons at a rate 10000 times higher, although the average brightness is only a factor of 100 times higher. The difference is entirely due to the difference in luminosity. The advantages of the Berkeley proposal, namely higher $N_{p}$ and smaller focussed spot size, are more than made up for by the much higher repetition rate of the IR Demo proposal. Because the IRFEL will normally operate in two-bunch mode, no additional hardware is needed to generate the Thomson scattered X-rays. Because the electron beam and optical beam are guaranteed to overlap (the electron beam is producing the optical beam after all!), many of the alignment and timing issues important in the $90^{\circ}$ scattering geometry vanish.

Compared to storage rings designed to produce light or $\mathrm{X}$-rays, the total fluxes are of modest average power. However, the pulse length of the emerging radiation is equal to the micropulse length, which can be very short for a linac beam. The bunch length reported above was from the current design parameters, driven somewhat by IRFEL commissioning needs. An opportunity exists to try to further reduce the bunch length. Given the rather modest chargeper-bunch of $135 \mathrm{pC}$ in our designs, compression to several hundred fsec may be possible, and will be explored.

\section{CONCLUSIONS}

In this paper formulas are given which completely describe the backscattered radiation for $180^{\circ}$ Thomson scattering. These results are applied to analyze a possible X-ray source based on the CW FELs being built/planned at Jefferson Lab. Even though the total brightness is not competitive with that generated by synchrotron light sources, to obtain short pulses $\left(\sigma_{t}<1 \mathrm{psec}\right)$ of X-rays, such an arrangement is probably superior. In turn, analyzing the short X-ray pulses may provide an excellent bunch-length diagnostic, particularly because the time of arrival of the X-rays will be known to better than $1 \mathrm{psec}$. The additional hardware needed to pursue this type of research at Jefferson Lab consists of X-ray handling and diagnostic equipment.

\section{ACKNOWLEDGEMENTS}

The author would like to thank K. Kim of the Berkeley laboratory for discussing the results at Berkeley with him, and for discussing why such a device might be interesting. G. Neil of Jefferson Lab was helpful with references to previous work in this field, and C. Bohn and H. F. Dylla provided encouragement to pursue this work. This work supported by U. S. DOE Contract No. DE-AC05-84ER40150.

\section{REFERENCES}

[1] Schoenlein, R. W., et. al., Science, 174, 236 (1996).

[2] Landau, L. D., and Lifshitz, E. M., Classical Theory of Fields, 4th Edition, Section 12.

[3] Jackson, J. D., Classical Electrodynamics, 2nd Edition, Section 14.7.

[4] Bohn, C. L., these Proceedings.

[5] Sprangle, P., Ting, A., Esarey, E., and Fisher, A., J. Appl. Phys., 725032 (1992). 